\title{
Meta-analysis of microRNAs as biomarkers for muscle-invasive bladder cancer
}

\author{
LIN-FENG ZHENG and WEN-YONG SUN
}

Department of Pathology, Zhejiang Cancer Hospital, Hangzhou, Zhejiang 310022, P.R. China

Received March 28, 2016; Accepted June 2, 2016

DOI: 10.3892/br.2016.705

\begin{abstract}
Bladder cancer is the most common cancer of the urinary tract. A quarter of bladder cancer patients presenting with muscle-invasive bladder cancer (MIBC) suffer significant morbidity and succumb to the disease. MicroRNA (miRNA) from tissue, urine or blood samples of MIBC patients have been demonstrated to differ from healthy individuals, and possibly have diagnostic value. The aim of the present meta-analysis was to access the overall diagnostic accuracy comprehensively and quantitatively. Systematic searching in PubMed, Web of Science, Embase and Chinese National Knowledge Infrastructure database was conducted. The pooled sensitivity, specificity, positive and negative likelihood ratios (PLR and NLR) and diagnostic odds ratio (DOR) were calculated via the random effects model to evaluate the overall test performance. Deeks' funnel plot asymmetry test was used to test the publication bias. A total of 10 studies were included in the meta-analysis, with a total of 577 patients and 412 controls. The pooled sensitivity and specificity were 0.78 [95\% confidence interval (CI), 0.69-0.86] and 0.77 (95\% CI, 0.72-0.81), respectively. The pooled PLR was 2.9 (95\% CI, 2.1-3.8), the NLR was 0.31 (95\% CI, 0.27-0.35), the DOR was 7 (95\% CI, 4-13) and the pooled AUC was 0.80 (95\% CI, 0.69-0.87). In conclusion, the current miRNA assays support their use as markers for MIBC diagnosis.
\end{abstract}

\section{Introduction}

Bladder cancer is one of the most common cancer ranking fourth in incidence in Western countries and first in

Correspondence to: Dr Lin-Feng Zheng, Department of Pathology, Zhejiang Cancer Hospital, 38 Guangji Road, Hangzhou, Zhejiang 310022, P.R. China

E-mail: zhenglf@zjcc.org.cn

Abbreviations: MIBC, muscle-invasive bladder cancer; NIBC, non-invasive bladder cancer; PLR, positive likelihood ratio; NLR, negative likelihood ratio; DOR, diagnostic odds ratio; SROC, summary receiver operator characteristic; SEN, sensitivity; SPE, specificity; AUC, area under SROC curve

Key words: meta-analysis, microRNA, muscle-invasive bladder cancer, biomarker, diagnosis
China $(1,2)$. There are $\sim 380,000$ new cases and 150,000 fatalities per year worldwide (3). Bladder cancer is staged via the tumor-node-metastasis system, which describes the extent of invasion (Tis-T4) (4,5). Approximately $75 \%$ of patients present with non-invasive bladder cancer (NIBC; stage Ta), but a quarter suffer muscle-invasive bladder cancer (MIBC) at the time of diagnosis (of stage T2 and above) and they have a less favorable prognosis with 5 -year survival $<50 \%(6,7)$. Despite improvements in surgical techniques and postoperative recovery pathways, this complex procedure remains highly challenging, and the treatment has not advanced for several decades $(8,9)$. Furthermore, the high cost of surgery and management of subsequent complications, chemotherapy, surveillance imaging and high end-of-life costs contribute to the substantial financial burden of advanced disease (10). Therefore, development of early biological markers for diagnosis of MIBC is of importance.

MicroRNA (miRNA) is endogenous RNA of 22 nucleotides that targets mRNA for cleavage or translational repression. miRNA families are responsible for a number of physiological processes, including cell growth/differentiation, maintenance of internal environmental stabilization, immune response and embryonic development of different cancers (11-14). Increasing evidence has suggested that miRNAs can be transmitted through extracellular vesicles, such as exosomes, that are devoted to cell-cell contact and influence, and additionally they are convenient for detection $(15,16)$. Thus far, various miRNAs have been identified to have prognostic values with bladder cancer, in NIBC and MIBC (17-19).

Currently, there are few meta-analyses published on the diagnostic performance of miRNA assays for MIBC. Therefore, the present study performed a meta-analysis to review and assess the overall diagnostic values of miRNA assays for MIBC.

\section{Materials and methods}

Publication search. To identify all the potentially eligible studies on miRNA polymorphisms and cancer risk, we carried out a systematic search on PubMed, Web of Science and Chinese National Knowledge Infrastructure, covering all studies published between January 1, 2000 and January 25, 2016, using the search terms: ('miR' or 'miRNAs' or 'microRNAs') and ('diagnostic value' or 'diagnoses' or 'receiver operating characteristics' or 'ROC curve' or 'sensitivity and specificity') 
and ('muscle-invasive bladder cancer, muscle-invasive urinary bladder neoplasms, muscle-invasive urothelial cancer, MIBC, MIUCC'). References of the retrieved studies and review studies were also screened. Qualified studies had to meet all the following standards: i) Diagnosis of MIBC in histology, ii) utility of miRNA expression profiles (from tissue or blood or urine) for urological cancers diagnosis. The exclusion criteria included: i) Reviews, case reports, and meta analyses, ii) studies not related to MIBC and the diagnostic value of miRNA for urological cancers, iii) studies without valid data.

Data extraction and quality assessment. Quality assessment was performed for each included study by independent reviewers using the Quality Assessment of Diagnostic Accuracy Studies (QUADAS-2) tool (20). The QUADAS-2 tool contains seven questions, and each one should be answered with 'yes' (1 score), 'unclear' or 'no' (0 score). All questions were given equal weight, resulting in a maximum possible score of 7. Conflicting evaluation was resolved following a full discussion.

The assessment consisted of four domains: Patient selection, index test, reference standard, and flow and timing. The first three domains were assessed in terms of applicability. Each of the four domains was assessed via the risk of bias. Assessments were labeled as 'high', 'low' or 'unclear', corresponding to high risk, low risk and unclear, respectively.

Statistical analysis. All the statistical analyses were performed using Rev Man 5.3 software (Copenhagen: The Nordic Cochrane Centre, The Cochrane Collaboration, 2014). The sensitivity and specificity data of miRNAs associated with the predicted and/or diagnostic value of MIBC were extracted from each study. First, the results of sensitivity, specificity, positive and negative likelihood ratio (PLR and NLR, respectively), diagnostic odds ratio (DOR) and $95 \%$ confidence intervals (CIs) were calculated using the random-effect model. Subsequently, the summary receiver operator characteristic (SROC) curve was created and the area under the SROC curve (AUC) was calculated. PLR was on behalf of the odds of positive test results of MIBC patients, while NLR reflected the odds of positive results in those without MIBC. DOR was the outcome of the combination of PLR and NLR (DOR = PLR/NLR). In addition, the heterogeneity between studies was evaluated through $\chi^{2}$ test and $\mathrm{I}^{2}$ test. If the tests show a $\mathrm{P}<0.1$ or $\mathrm{I}^{2}>50 \%$, the existence of significant heterogeneity would be verified (21). Subsequently, meta-regression and subgroup analyses were undertaken to explore the sources of between-study heterogeneity. Furthermore, Deeks' funnel plots were adopted to evaluate the publication bias.

The percentages of patients in each subgroup were calculated for the categorical variables using unpaired Student's t-test, $\chi^{2}$ test or Fisher's exact test, appropriately.

\section{Results}

Patient characteristics. The flow graph of study selections is shown in Fig. 1. A total of 125 potentially relevant studies were selected with an established search strategy. Following a detailed evaluation, 10 studies (22-31) were used for the

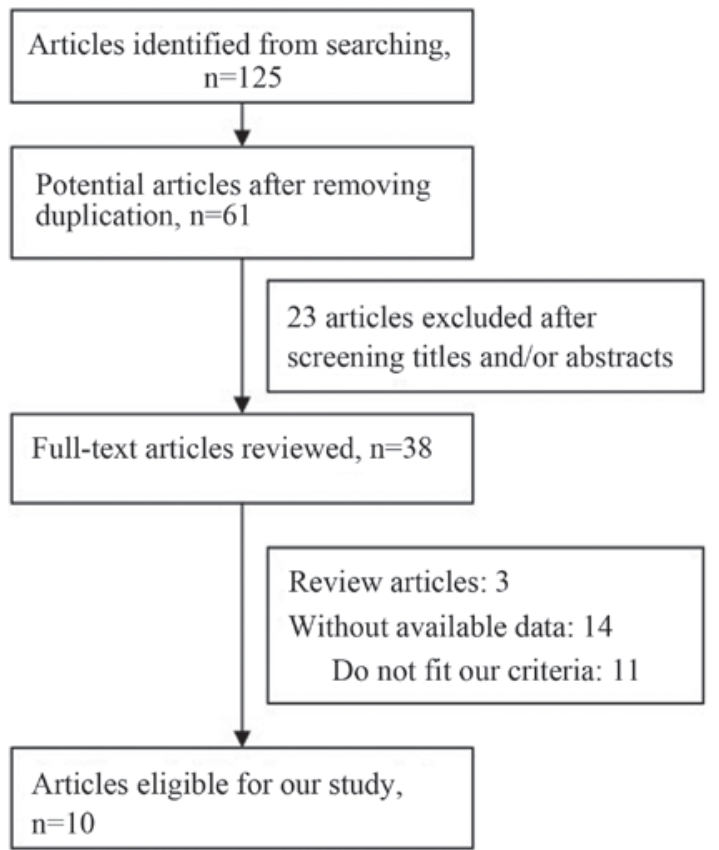

Figure 1. Flow chart of the included studies.

meta-analysis. The main characteristics of the included studies are summarized in Table I. Among the 10 studies, the total number of patients and controls were 577 and 412, respectively. Four studies were conducted in Asian populations, while the remaining studies were conducted in Caucasian populations. The diagnostic performances of single and multiple miRNAs have been investigated among those included studies.

Quality assessment of studies. The results of the QUADAS-2 assessment are shown as a bar graph in Fig. 2. The majority of all included studies fulfilled the majority of the items in QUADAS-2, which indicated that the general quality of the included studies is good.

Diagnostic accuracy. Due to the existence of significant heterogeneity between studies in sensitivity and specificity $\left(\mathrm{I}^{2}=81.3 \%\right.$ and $\left.\mathrm{I}^{2}=77.8 \%\right)$, the random effects model was adopted. Fig. 3 depicts the forest plots of data from the miRNA panels subgroup and mean sensitivity and specificity. The pooled results of diagnostic criteria and their $95 \%$ CIs are listed in Table II. The overall sensitivity, specificity, PLR, NLR and DOR were 0.78 (0.69-0.86), 0.77 (0.72-0.81), 2.9 (95\% CI, 2.1-3.8), 0.31 (95\% CI, 0.27-0.35), 7 (95\% CI, 4-13), respectively. Moreover, the SROC curve was generated and the AUC was calculated as 0.80 (95\% CI, 0.69-0.87) (Fig. 4), which implied a relatively high diagnostic accuracy.

Subgroup analyses. A subgroup analyses was also performed to identify potential sources of heterogeneity (Table II). For single miRNA profiling assays, sensitivity, specificity and AUC values were 0.70 (0.65-0.74), 0.73 (95\% CI, 0.67-0.79), and 0.79 (95\% CI, 0.76-0.82), respectively. For multiple miRNAs, the sensitivity (SEN), specificity (SPE) and AUC values were 0.81 (95\% CI, 0.72-0.91), 0.84 (95\% CI, 0.75-0.93) and 0.86 (95\% CI, 0.83-0.89), respectively. These data indicated that multiple miRNAs profiling were more accurate 


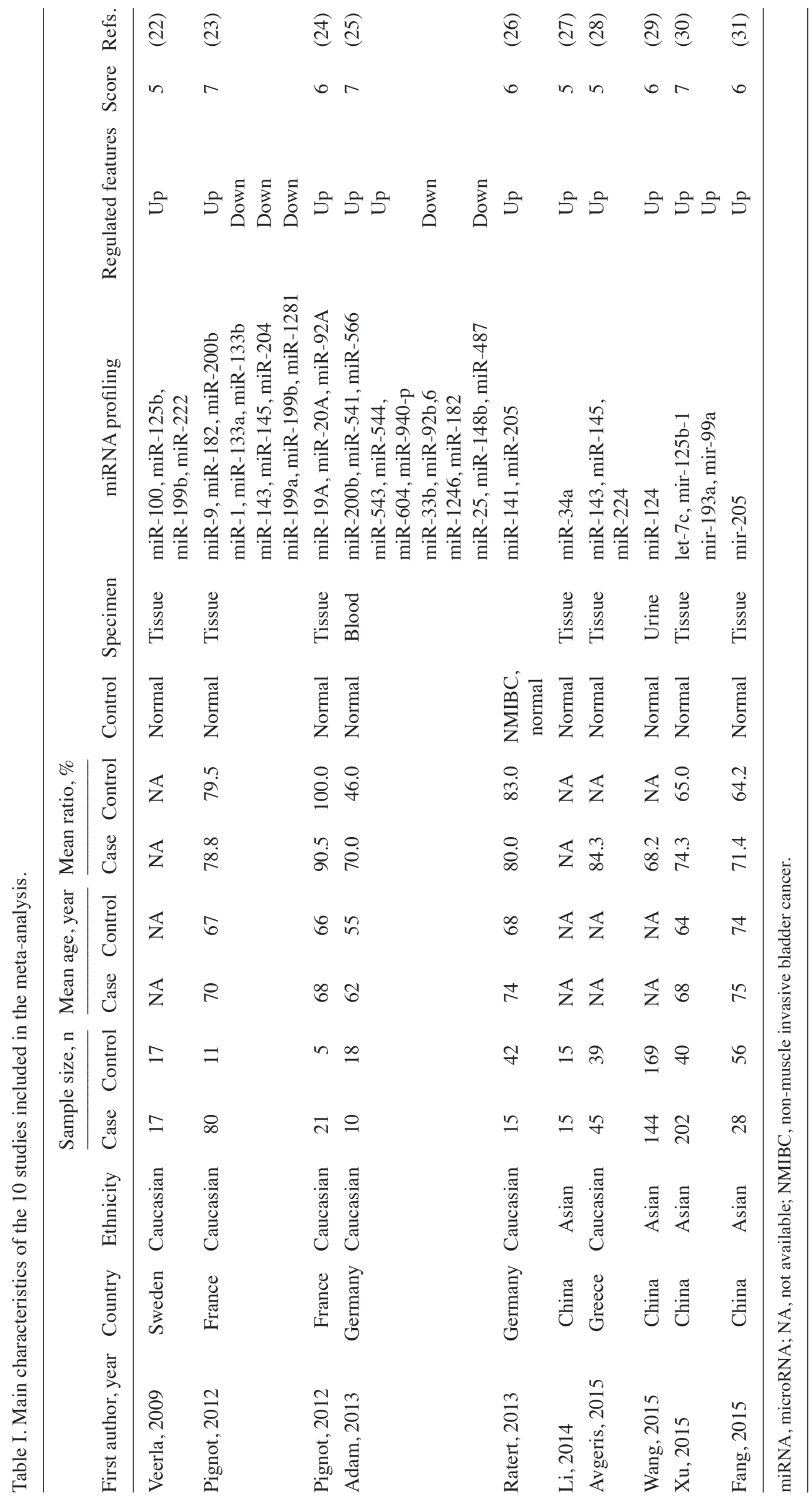


Table II. Summary estimates of diagnostic criteria and the $95 \%$ confidence intervals (CIs).

\begin{tabular}{lcccccc}
\hline Analysis & $\begin{array}{c}\text { Sensitivity } \\
(95 \% \mathrm{CI})\end{array}$ & $\begin{array}{c}\text { Specificity } \\
(95 \% \mathrm{CI})\end{array}$ & $\begin{array}{c}\text { PLR } \\
(95 \% \mathrm{CI})\end{array}$ & $\begin{array}{c}\text { NLR } \\
(95 \% \mathrm{CI})\end{array}$ & $\begin{array}{c}\text { DOR } \\
(95 \% \mathrm{CI})\end{array}$ & $\begin{array}{c}\text { AUC } \\
(95 \% \mathrm{CI})\end{array}$ \\
\hline $\begin{array}{l}\text { Ethnicity } \\
\text { Caucasian }\end{array}$ & $0.71(0.66-0.75)$ & $0.76(0.67-0.83)$ & $2.4(2.0-2.8)$ & $0.34(0.22-0.46)$ & $6(4-11)$ & $0.74(0.69-0.78)$ \\
$\quad$ Asian & $0.67(0.63-0.72)$ & $0.75(0.73-0.82)$ & $2.5(2.1-3.0)$ & $0.32(0.21-0.42)$ & $7(5-12)$ & $0.82(0.77-0.86)$ \\
miRNA profiling & & & & & & \\
$\quad \begin{array}{l}\text { Single miRNA } \\
\text { Multiple miRNA }\end{array}$ & $0.70(0.65-0.74)$ & $0.73(0.67-0.79)$ & $2.6(2.3-2.9)$ & $0.38(0.29-0.47)$ & $5(3-10)$ & $0.79(0.76-0.82)$ \\
Overall & $0.81(0.72-0.91)$ & $0.84(0.75-0.93)$ & $4.2(2.7-5.7)$ & $0.26(0.15-0.37)$ & $16(9-24)$ & $0.86(0.83-0.89)$ \\
& $0.78(0.69-0.86)$ & $0.77(0.72-0.81)$ & $2.9(2.1-3.8)$ & $0.31(0.27-0.35)$ & $7(4-13)$ & $0.80(0.69-0.87)$ \\
\hline
\end{tabular}

PLR, positive likelihood ratio; NLR, negative likelihood ratio; DOR, diagnostic odds ratio; AUC, area under the curve; miRNA, microRNA.

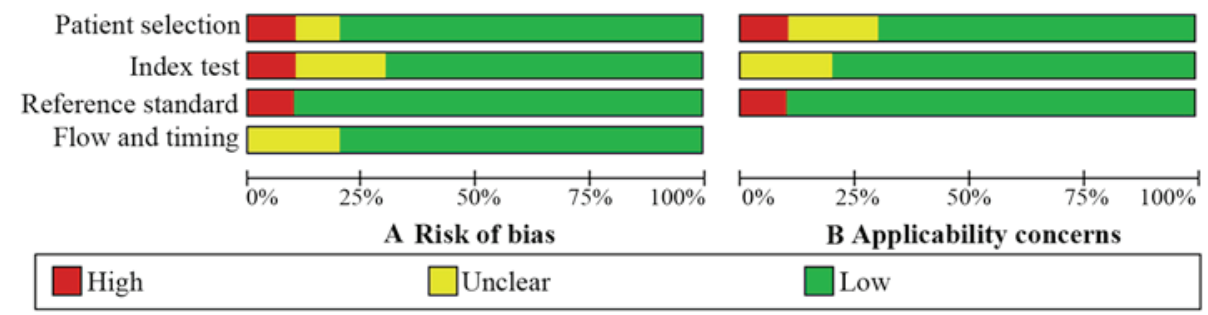

Figure 2. General assessment quality of the included studies using the Quality Assessment of Diagnostic Accuracy Studies-2 tool.

A Study
Adam L, et al 2013
Avgeris $M$, et al 2015
Heng li, et al 2014
Jiang XM, et al 2015
Pignot $G$, Vieillefond $A$, et al 2012
Pignot $G$, Cizeron-Clairac $G$, et al 2012
Ratert $N$, et al 2013
Veerla $S$, et al 2009
Wang JF, et al 2015
Xu Z, et al 2015

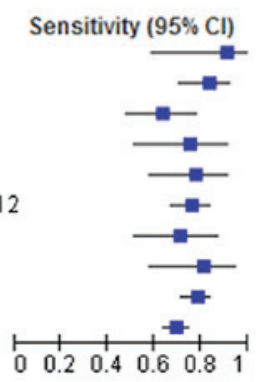

Sensitivity

B Study

Adam L, et al 2013

Avgeris M et al 2015

Heng li, et al 2014

Jiang XM, et al 2015

Pignot $G$, Vieillefond $A$, et al 2012

Pignot $G$,Cizeron-Clairac $G$, et al 2012

Ratert $N$, et al 2013

Veerla $S$, et al 2009

Wang JF, et al 2015

$\mathrm{XuZ}$, et al 2015

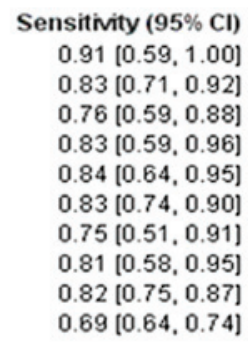

Specificity $(95 \% \mathrm{Cl})$

$0.60[0.32,0.84]$

$0.74[0.54,0.89]$

$0.82[0.48,0.98]$

$0.70[0.35,0.93]$

$0.67[0.35,0.90]$

$0.81[0.65,0.92]$

$0.83[0.52,0.98]$

$0.86[0.65,0.97]$

$0.79[0.49,0.95]$

$0.75[0.62,0.85]$

Specificity

Figure 3. Forest plots of (A) sensitivity and (B) specificity of miRNAs for the miRNA panel subgroups. CI, confidence interval.

than single miRNA profiling. The studies based on Caucasian populations had a pooled sensitivity of 0.71 and a specificity of 0.76 , while studies based on Asian populations had a relatively lower sensitivity (0.67) and specificity (0.75). The subgroup analyses suggested that the ethnicity and miRNA profiling had an evident influence on the diagnostic accuracy. 


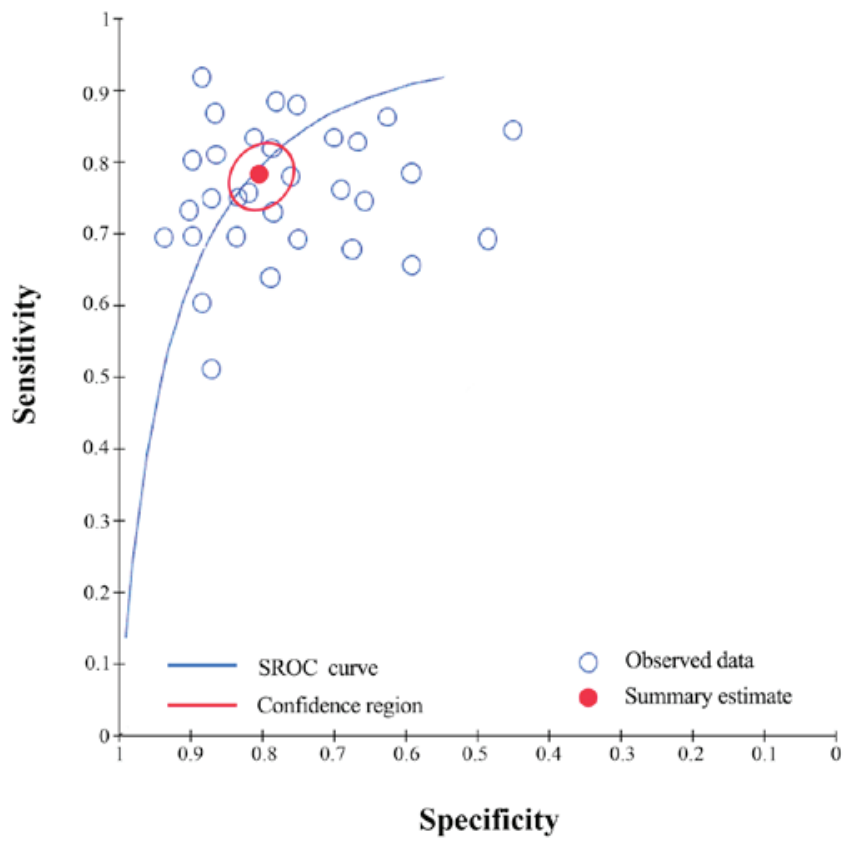

Figure 4. SROC curve of the studies with confidence and prediction region around mean operating sensitivity and specificity. SROC, summary receiver operator characteristic.

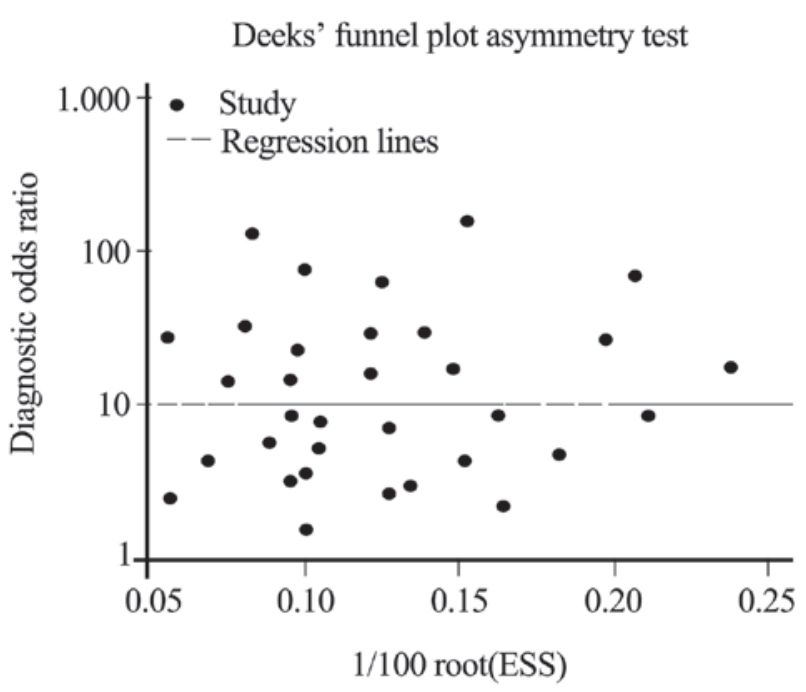

Figure 5. Deeks' linear regression test of funnel plot asymmetry. ESS, effective sample size.

Therefore, these factors may be the potential sources of heterogeneity.

Publication bias. Finally, the Deeks' funnel plot asymmetry test was conducted to evaluate the publication bias. The P-value of 0.7 suggested that no significant publication bias existed among the studies (Fig. 5).

\section{Discussion}

Although clear progression in surgery and chemical treatment has been achieved in MIBC, the prognosis of the patients is poor $(6,7)$. As aforementioned, approximately a quarter of bladder cancer patients have MIBC at diagnosis, and the critical factor is how to detect and diagnose MIBC as early as possible. Thus, novel and reliable biomarkers for MIBC detection are urgently required.

As the existing biomarkers do not exhibit high sensitivity and specificity in MIBC detection, miRNAs have been reported as markers of MIBC in numerous studies. Pignot et al (23) reported that miR-9, miR-182 and miR-200b were associated with MIBC aggressiveness, and with recurrence-free and overall survival in univariate analysis and multivariate analysis. Avgeris et al (28) reported that high miR-143/145 levels could predict the inferior overall survival for MIBC effectively and the progression of superficial tumors independently. Xu et al (30) identified four specific miRNAs (let-7c, mir-125b-1, mir-193a, and mir-99a) in association with the progression and aggressiveness of MIBC via microarray analysis. Several genome-wide profiling studies have been reported and identified specific miRNA alterations in bladder cancer (32-34). Those results suggested a promising prognostic value of these miRNAs markers.

However, as the association between miRNAs and MIBC are inconsistent and the studies are designed differently, comparing the wide ranges of diagnostic performance is difficult. Therefore, the present study aimed to summarize the result of individual studies and investigate the diagnostic value of miRNAs for MIBC.

Following analysis, the overall sensitivity and specificity of miRNAs was 0.78 and 0.77 , which indicated accuracy of miRNAs to detect MIBC. The pooled PLR was 2.9 and the pooled NLR was 0.31, respectively. The AUC was 0.80 and DOR was 7. These data suggested that miRNAs had a relatively high diagnostic accuracy. As heterogeneity between studies could affect the results of the meta-analysis, subgroup analyses will aid to understand these influences. Therefore, subgroup analyses were performed based on ethnicity and miRNA profiling. Subgroups of miRNA profiling indicated that multiple miRNA assays (SEN, SPE and AUC of 0.81 , 0.84 and 0.86 , respectively) had a higher diagnostic performance than those of single miRNA assays (SEN, SPE and AUC of $0.70,0.73$ and 0.79 , respectively). No significant different was observed for the miRNA expression profile test between the Asian and Caucasian groups.

There were several limitations in the meta-analysis. First, the included studies were based on limited sample size, and if the specimen of miRNA could be divided into blood, urine, tissue groups, do analysis and comparison respectively, the results may be more significant and accurate. Second, the majority of the included studies did not differentiate the grade of MIBC, and therefore, subgroup analyses based on these variables were restricted due to limited reported data. Third, as the majority of the studies did not provide further miRNA research, the changes of miRNA in different stages of MIBC, following treatment in different methods or in patients who received treatment experiencing MIBC again could not be verified.

In conclusion, the present study analyzed the pooled data of SEN, SPE, PLR, NLR, DOR and AUC from 10 studies. miRNA assays could serve as markers for MIBC diagnosis, particularly the combined usage of miRNA, and have a good potential as an accurate biomarker to diagnose of MIBC. However, the clinical application of miRNA profiling for MIBC diagnosis remains validating in future studies. 


\section{References}

1. Knowles MA and Hurst CD: Molecular biology of bladder cancer: New insights into pathogenesis and clinical diversity. Nat Rev Cancer 15: 25-41, 2015.

2. Xu S, Zhang GM, Guan FJ, Dong DH, Luo L, Li B, Ma XC, Zhao $J$ and Sun LJ: The association between metabolic syndrome and the risk of urothelial carcinoma of the bladder: A case-control study in China. World J Surg Oncol 13: 236, 2015.

3. Ferlay J, Shin HR, Bray F, Forman D, Mathers C and Parkin DM: Estimates of worldwide burden of cancer in 2008: GLOBOCAN 2008. Int J Cancer 127: 2893-2917, 2010.

4. Rocken $\mathrm{C}$ and Behrens HM: Validating the prognostic and discriminating value of the TNM-classification for gastric cancer - a critical appraisal. Eur J Cancer 51: 577-586, 2015.

5. Eble JN, Sauter G, Epstein JI and Sesterhen IA (eds): World Health Organization Classification of Tumours. Pathology and genetics of tumours of the urinary system and male genital organs. IARC Press, Lyon, 2004.

6. Soloway MS: Bladder cancer: Lack of progress in bladder cancer - what are the obstacles? Nat Rev Urol 10: 5-6, 2013.

7. Chamie K and Litwin MS: Quality of bladder cancer care in the USA. Expert Rev Pharmacoecon Outcomes Res 11: 619-621, 2011.

8. Corcoran AT, Handorf E, Canter D, Tomaszewski JJ, Bekelman JE, Kim SP, Uzzo RG, Kutikov A and Smaldone MC: Variation in performance of candidate surgical quality measures for muscle-invasive bladder cancer by hospital type. BJU Int 115 : 230-237, 2015.

9. Jayaratna IS, Navai N and Dinney CP: Risk based neoadjuvant chemotherapy in muscle invasive bladder cancer. Transl Androl Urol 4: 273-282, 2015

10. Johnson DC, Greene PS and Nielsen ME: Surgical advances in bladder cancer: At what cost? Urol Clin North Am 42: 235-252, ix, 2015.

11. Bartel DP: MicroRNAs: Genomics, biogenesis, mechanism, and function. Cell 116: 281-297, 2004.

12. Beitzinger M and Meister G: Preview. MicroRNAs: From decay to decoy. Cell 140: 612-614, 2010.

13. Calin GA and Croce CM: MicroRNA signatures in human cancers. Nat Rev Cancer 6: 857-866, 2006.

14. Kent OA and Mendell JT: A small piece in the cancer puzzle: MicroRNAs as tumor suppressors and oncogenes. Oncogene 25 6188-6196, 2006.

15. Maida Y, Takakura M, Nishiuchi T, Yoshimoto T and Kyo S: Exosomal transfer of functional small RNAs mediates cancer-stroma communication in human endometrium. Cancer Med 5: 304-314, 2016

16. Kim MS, Haney MJ, Zhao Y, Mahajan V, Deygen I, Klyachko NL, Inskoe E, Piroyan A, Sokolsky M, Okolie O, et al: Development of exosome-encapsulated paclitaxel to overcome MDR in cancer cells. Nanomedicine 12: 655-664, 2016

17. Catto JW, Miah S, Owen HC, Bryant H, Myers K, Dudziec E, Larré S, Milo M, Rehman I, Rosario DJ, et al: Distinct microRNA alterations characterize high- and low-grade bladder cancer. Cancer Res 69: 8472-8481, 2009.

18. Pignot G, Cizeron-Clairac G, Vacher S, Susini A, Tozlu S, Vieillefond A, Zerbib M, Lidereau R, Debre B, Amsellem-Ouazana D, et al: MicroRNA expression profile in a large series of bladder tumors: Identification of a 3-miRNA signature associated with aggressiveness of muscle-invasive bladder cancer. Int J Cancer 132: 2479-2491, 2013.

19. Segersten U, Spector Y, Goren Y, Tabak S and Malmström PU: The role of microRNA profiling in prognosticating progression in Ta and T1 urinary bladder cancer. Urol Oncol 32: 613-618, 2014.

20. Whiting PF, Rutjes AW, Westwood ME, Mallett S, Deeks JJ, Reitsma JB, Leeflang MM, Sterne JA and Bossuyt PM; QUADAS-2 Group: QUADAS-2: A revised tool for the quality assessment of diagnostic accuracy studies. Ann Intern Med 155: $529-536,2011$
21. Dinnes J, Deeks J, Kirby J and Roderick P: A methodological review of how heterogeneity has been examined in systematic reviews of diagnostic test accuracy. Health Technol Assess 9: 1-113, iii, 2005.

22. Veerla S, Lindgren D, Kvist A, Frigyesi A, Staaf J, Persson H, Liedberg F, Chebil G, Gudjonsson S, Borg A, et al: miRNA expression in urothelial carcinomas: Important roles of miR-10a, miR-222, miR-125b, miR-7 and miR-452 for tumor stage and metastasis, and frequent homozygous losses of miR-31. Int J Cancer 124: 2236-2242, 2009.

23. Pignot G, Cizeron-Clairac G, Vacher S, Susini A, Tozlu S, Zerbib M, Lidereau R, Debre B, Amsellem-Ouazana D and Bieche I: MicroRNA expression profile in a large series of bladder tumors: Identification of a 3-miRNA signature predictive of aggressiveness and prognosis of muscle-invasive bladder cancer. Eur Urol Suppl 11: e169, 2012.

24. Pignot G, Vieillefond A, Vacher S, Zerbib M, Debre B, Lidereau R, Amsellem-Ouazana D and Bieche I: Hedgehog pathway activation in human transitional cell carcinoma of the bladder. Br J Cancer 106: 1177-1186, 2012.

25. Adam L, Wszolek MF, Liu CG, Jing W, Diao L, Zien A Zhang JD, Jackson D and Dinney CP: Plasma microRNA profiles for bladder cancer detection. Urol Oncol 31: 1701-1708, 2013.

26. Ratert N, Meyer HA, Jung M, Lioudmer P, Mollenkopf HJ, Wagner I, Miller K, Kilic E, Erbersdobler A, Weikert S, et al: miRNA profiling identifies candidate mirnas for bladder cancer diagnosis and clinical outcome. J Mol Diagn 15: 695-705, 2013.

27. Li H, Yu G, Shi R, Lang B, Chen X, Xia D, Xiao H, Guo X, Guan W, Ye Z, et al: Cisplatin-induced epigenetic activation of miR-34a sensitizes bladder cancer cells to chemotherapy. Mol Cancer 13: 8, 2014.

28. Avgeris M, Mavridis K, Tokas T, Stravodimos K, Fragoulis EG and Scorilas A: Uncovering the clinical utility of miR-143, miR-145 and miR-224 for predicting the survival of bladder cancer patients following treatment. Carcinogenesis 36: 528-537, 2015.

29. Wang J, Zhang X, Wang L, Dong Z, Du L, Yang Y, Guo Y and Wang C: Downregulation of urinary cell-free microRNA-214 as a diagnostic and prognostic biomarker in bladder cancer. J Surg Oncol 111: 992-999, 2015.

30. Xu Z, Yu YQ, Ge YZ, Zhu JG, Zhu M, Zhao YC, Xu LW, Yang XB, Geng LG, Dou QL, et al: MicroRNA expression profiles in muscle-invasive bladder cancer: Identification of a four-microRNA signature associated with patient survival. Tumour Biol 36: 8159-8166, 2015.

31. Fang Z, Dai W, Wang X, Chen W, Shen C, Ye G and Li L: Circulating miR-205: A promising biomarker for the detection and prognosis evaluation of bladder cancer. Tumour Biol 37: 8075-8082, 2016

32. Catto JW, Abbod MF, Wild PJ, Linkens DA, Pilarsky C, Rehman I, Rosario DJ, Denzinger S, Burger M, Stoehr R, et al: The application of artificial intelligence to microarray data: Identification of a novel gene signature to identify bladder cancer progression. Eur Urol 57: 398-406, 2010.

33. Silva-Santos RM, Costa-Pinheiro P, Luis A, Antunes L, Lobo F, Oliveira J, Henrique R and Jerónimo C: MicroRNA profile: A promising ancillary tool for accurate renal cell tumour diagnosis. Br J Cancer 109: 2646-2653, 2013.

34. Jiang X, Du L, Wang L, Li J, Liu Y, Zheng G, Qu A, Zhang X, Pan H, Yang Y, et al: Serum microRNA expression signatures identified from genome-wide microRNA profiling serve as novel noninvasive biomarkers for diagnosis and recurrence of bladder cancer. Int J Cancer 136: 854-862, 2015. 